\title{
Dégradabilité en sachets des matières azotées des aliments concentrés : standardisation de la méthode et variabilités intra- et interlaboratoires
}

\author{
R Vérité1, B Michalet-Doreau², F Vedeau ${ }^{3}$, P Chapoutot ${ }^{4}$ \\ 1 INRA, Station de Recherches sur la Vache Laitière, 35590 L'Hermitage; 2 INRA, Theix, \\ Unité de la Valeur des Aliments, 63122 Ceyrat; ${ }^{3}$ Sanders, 17, quai de I'Industrie, BP 32 , \\ 91200 Athis Mons Cedex; ${ }^{4}$ INRA, INA-PG, Station de Nutrition et Alimentation, \\ 16, rue C-Bernard, 75231 Paris Cedex 05, France
}

Summary - In sacco degradability of feed protein (DT) was measured in 13 feedstuffs by 4 laboratories according the French standardized procedure. Adjustment for variations in microbial activity by reference to DM disappearance of a standard sample of lucerne decreased within and between lab variability. When the standardized procedure was carefully followed, results were in rather good agreement between labs.

Introduction - Pour diminuer la variabilité de la mesure in sacco de la dégradabilité de l'azote alimentaire (DT) la méthode a été standardisée en France dans le cadre du système des protéines digestibles dans l'intestin (PDI) (MichaletDoreau et al, 1987). Nous avons testé la reproductibilité de la méthode entre laboratoires et comparé l'intérêt de différents échantillons de référence pour prendre en compte les fluctuations de l'activité microbienne dans le rumen.

Matériel et Méthodes - La méthode décrite par Michalet-Doreau et al (1987) a été appliquée par chacun des 4 laboratoires ( $c f$ auteurs) sur les 13 aliments du tableau I à raison de 3 répétitions par lieu (= 3 vaches), chaque répétition étant constituée par 2 séries de 6 points $(2,4,8,16,24$ et $48 \mathrm{~h})$ passées 2 j successifs. Chaque série de mesures comportait aussi 4 sachets de référence contenant de la luzerne déshydratée (retrait après 8 ou $16 \mathrm{~h}$ ) ou du tourteau de soja (retrait après 2 ou $4 \mathrm{~h}$ ). Les échantillons ont été fournis broyés à la grille de $0,8 \mathrm{~mm}$. Le calcul de la dégradation théorique (DT) a été effectué par la méthode séquentielle.
Résultats et Discussion - Sachets de référence et précision. Les valeurs DT observées présentent des différences significatives entre lieux et entre vaches dans certains laboratoires. Ces variations sont liées à celles du taux de disparition de la MS dans les sachets de luzerne de référence à $8 \mathrm{~h}$ (dl 8 ), les autres sachets de référence n'apportent rien en complément de $d / 8$ [DT obs $=f$ (aliments) $+0,579$ $x$ dl $8 ;$ syx $=2.46]$. dl 8 rend compte de $43 \%$ des variations de DT intraaliment et diminue fortement ou annule les différences interlieu et intervache. Des résultats antérieurs (Vérité et Michalet, non publié), obtenus dans les laboratoires 1 et 2 sur 199 aliments (502 mesures) avaient donné la même relation [DT $=f($ alim) + $0,582 \times$ dl 8 avec $R^{2}=0,44$ et $s=1,48$ ] et avaient conduit à une procédure de standardisation que nous proposons de maintenir : $\mathrm{DT}_{\text {ajustée }}=\mathrm{DT}_{\text {obs }}-0,6 \times(d / 8$ -45); où 45 représente la valeur standard de dl 8 (moyenne des premières mesures). 
La précision de la mesure (variabilité entre triple) est ainsi améliorée (passant de 2,2 à 1,7 pts) et assez voisine entre les 4 laboratoires (entre 1,4 et 2,0 pts). Elle diffère peu entre aliments (entre 1,0 et 1,6 pts) sauf pour les pulpes de betterave $(3,6$ pts).

Reproductibilité entre laboratoires. Les valeurs de $\mathrm{DT}_{\text {ajustée }}$ sont très cohérentes entre les 4 laboratoires pour 8 aliments (tableau I) (écart type entre laboratoires compris entre 1,0 et 2,0 pts); par contre, l'accord est moins bon pour les 5 autres aliments (luzerne, colza, maïs, pulpe, orge). En fait, les laboratoires 1 et 2 sont en très bon accord sur tous les aliments (écart type des différences = 1,2 pt). Le laboratoire $D$ donne des valeurs supérieures en moyenne de 1,5 pt; ce léger biais pourrait provenir du trempage préalable de 15 min des sachets effectué par erreur dans ce laboratoire. Le laboratoire $\mathrm{C}$ est en bon accord sur 8 aliments, mais donne des écarts assez larges et fluctuants sur les 5 autres aliments ( -5 à -11 pts); ce laboratoire a dû changer de technique à l'occasion de ce travail et pouvait manquer d'habitude sur la méthode standard.

La répétabilité de la mesure est bonne entre les laboratoires sous réserve d'une observation rigoureuse de toutes les conditions de mesure. Les sachets de luzerne de référence prennent bien en compte les variations d'activité microbienne d'origines variées (lieu, vache, jour) l'ajustement est donc important à effectuer.

Michalet-Doreau $B$, Vérité $R$, Chapoutot $P$ (1987) Bull Tech CRZV Theix, INRA 69, 5-7

Tableau I. Variations entre laboratoires de la DT (valeurs ajustées, A-D) des protéines des aliments.

\begin{tabular}{|c|c|c|c|c|c|c|c|}
\hline & $A$ & $B$ & $c$ & $D$ & $\begin{array}{l}\text { Valeur } \\
\text { moyenne }\end{array}$ & $\begin{array}{l}\text { Préci- } \\
\text { sion a }^{-}\end{array}$ & $\begin{array}{l}\text { Reproduc- } \\
\text { tibilité }^{b}\end{array}$ \\
\hline Son de blé & 82,9 & 80,9 & 80,0 & 82,3 & 81,5 & 1,2 & 1,3 \\
\hline Luzerne déshydratée & 66,3 & 64,9 & 56,0 & 71,1 & 64,6 & 1,0 & 6,3 \\
\hline Tourteau de soja & 74,2 & 70,8 & 71,7 & 71,1 & 72,0 & 1,6 & 1,5 \\
\hline Farine de viande & 57,3 & 55,3 & 57,1 & 60,0 & 57,4 & 1,4 & 1,9 \\
\hline Tourteau de colza & 43,1 & 45,7 & 39,9 & 49,6 & 44,6 & 1,1 & 4,1 \\
\hline Maïs & 42,7 & 43,4 & 37,6 & 46,2 & 42,5 & 1,3 & 3,6 \\
\hline Tourteau de tournesol & 79,5 & 77,4 & - & 75,3 & 77,4 & 1,4 & 2,1 \\
\hline Tourteau d'arachide & 74,9 & 74,9 & 74,2 & 76,7 & 75,2 & 1,5 & 1,1 \\
\hline Corn gluten meal & 32,4 & 33,9 & 34,8 & 35,7 & 34,2 & 1,5 & 1,4 \\
\hline Tourteaux tannés & 33,2 & 36,5 & 33,5 & 33,0 & 34,1 & 1,4 & 1,6 \\
\hline Pois & 94,1 & 94,2 & 94,9 & 92,5 & 93,9 & 1,4 & 1,0 \\
\hline Pulpes de betterave & 63,4 & 59,8 & 47,3 & 63,6 & 58,5 & 3,6 & 7,7 \\
\hline Orge & 85,9 & 86,4 & 79,0 & 88,9 & 85,1 & 1,4 & 4,2 \\
\hline Moyenne & 63,8 & 63,4 & 58,8 & 65,1 & & & \\
\hline Précision a & 1,4 & 2,0 & 1,6 & 1,7 & & & \\
\hline
\end{tabular}

$a^{a}$ Ecart type entre triple (intraaliment et intralieu); moyenne par aliment ou par lieu. ${ }^{b}$ Ecart type entre laboratoires pour un même aliment. 